\title{
DRB and DCCB: Efficient and Robust Dynamic Broadcast for Ad Hoc and Sensor Networks
}

\author{
Alireza Keshavarz-Haddad ${ }^{\dagger} \quad$ Vinay Ribeiro $§ \quad$ Rudolf Riedi ${ }^{\dagger}$ \\ Department of Electrical and Computer Engineering $\dagger$, Department of Statistics $\ddagger$ \\ Rice Houston, University, TX 77005, USA \\ Department of Computer Science and Engineering, I.I.T. Delhi, New Delhi -110016, INDIA $\S$
}

\begin{abstract}
Deterministic, timer-based broadcast schemes not only guarantee full reachability over an idealistic lossless MAC layer, they also stand out for their robustness against node failure as well as more general changes in the network topology. This paper proposes the first broadcast schemes in this class which provably perform within a factor of the optimal efficiency (in terms of number of rebroadcasts). To the best of our knowledge no other deterministic timer-based scheme possesses this property. NS-2 simulations employing the 802.11b MAC protocol confirm our analysis. The factor can be estimated to be quite small.

Novel to the proposed schemes is also their hybrid backbone consisting of a given, static Dominating Set (DS) and a dynamically computed set of connecting nodes. As an additional contribution, this paper studies the trade-off of timer settings (and thus latency) against the number of rebroadcasts, as well as the robustness of the proposed algorithms. To the best of our knowledge, no prior study of these issues in the context of broadcast exists.
\end{abstract}

\section{INTRODUCTION}

In ad hoc networks, broadcast plays a particularly important role, relaying a message generated by one node to all other nodes. Broadcast is an integral part of a variety of protocols that provide basic functionality and efficiency to higher-layer services. Examples include coordinated and distributed computing, a prime task in sensor networks, and Ad Hoc unicast routing protocols such as DSR, AODV, ZRP, and LAR [1].

One simple approach for broadcasting is blind flooding, where each node rebroadcasts a packet as soon as it receives it for the first time. While simple and effective, blind flooding also produces redundant broadcasts and wastes precious bandwidth and power. These detrimental effects become particularly dramatic in dense networks, where blind flooding leads to the so-called broadcast storm which manifests in heavy contention and collisions (see the pioneering work of [2]).
Broadcast algorithms aim at avoiding the broadcast storm by forwarding a broadcast only over a subset of nodes called backbone. The performance of such a broadcast algorithm is usually measured by three metrics: efficiency measured in number of rebroadcasts reflects directly on the bandwidth and power consumption, reachability reports the fraction of nodes that actually receive the broadcast packet, and latency indicates the time between first transmission and the first time the last node in the network received the broadcast.

The most common classification [3], [4] labels a broadcast scheme deterministic if it guarantees full reachability assuming no loss at the MAC/PHY layer (for examples see [5]-[18]). All other schemes are called probabilistic (for examples see [2], [19]-[22]). Also, broadcast schemes are divided into static ones, that always use the same backbone and dynamic ones that recompute a backbone for each broadcast in order to adapt to changing network topology and broadcast state [13]. An important class of dynamic schemes is formed by the timer-based schemes where each network node starts a random timer upon hearing the broadcast and deciding upon timer expiration whether to rebroadcast based on the information collected from all overheard broadcast packets. Timer-based schemes include most probabilistic schemes (for an overview see [2], [21], [22]) as well as a few deterministic schemes (see [6], [10], [11]). A recent scheme uses non-random timers which depend on neighbor locations [23].

In this paper we concentrate on deterministic timerbased schemes, a class with attractive properties. By definition, their reachability is ideal assuming no loss at the MAC layer. In addition, being dynamic they are adaptive to network conditions and should be expected to be more robust to node failure and mobility than static schemes. As a result of their random timers, however, timer-based schemes incur a larger latency when compared to other schemes. 
The contribution of this paper is two-fold. First, we introduce two novel deterministic timer-based schemes: Dynamic Reflector Broadcast (DRB) and Dynamic Connector-Connector Broadcast (DCCB); both possess an efficiency within a factor of the optimum, a property which other deterministic timer-based schemes do not share and which we establish both analytically and in simulation. Second, we study by simulation the effect of the settings of the random timer for existing and proposed deterministic timer-based schemes. To the best of our knowledge, this issue has not been addressed in the literature before. We summarize the paper as follows.

The principal distinguishing feature of DRB and DCCB from other existing timer-based schemes is their hybrid backbone consisting of a fixed and a variable part. The fixed set of backbone nodes forms a Dominating Set (DS), i.e., it covers the entire network provided no loss occurs on the MAC/PHY layer. Hence, both algorithms are deterministic. The fixed DS is assumed to be given and efficient. The algorithms select the variable portion of the backbone nodes which have the task to connect the fixed given DS; they are selected randomly based on timers and overheard information; hence, both algorithms are timer-based.

The efficiency in terms of rebroadcasts of both schemes lies within a small factor of the optimum as our analysis shows. To allow for flexibility, DCCB assumes nothing about the DS. To gain yet another factor of efficiency, on the other hand, DRB assumes that the DS actually forms a Weakly Connected Dominating Set (WCDS), meaning that connecting all of its members which are within two hops of each other produces a Connected Dominating Set (CDS). Several algorithms for building an efficient WCDS or DS in a distributed fashion are known to exist [7], [24]-[28].

Our simulations with NS-2.29 [29] confirm our analytical findings regarding efficiency. Furthermore, they reveal the superior robustness to node failure of DCCB and DRB in terms of reachability. We suggest that robustness is a direct consequence of the hybrid nature of the backbone which allows for adaptively replacing nodes of the variable part that are no longer available. Efficiency, on the other hand, results from a clever scheme for connecting the given DS inherent to DCCB and DRB. Thereby, simulation indicates that DRB is particularly efficient, and DCCB particularly robust.

The effect of timer settings on performance has found only little attention in the literature so far. By simulation we find that the duration of the random timers controls a trade-off between the efficiency (number of rebroadcasts) and the latency for all timer-based schemes. Large timer averages increase the latency but reduce the number of rebroadcasts, while small timer averages show the opposite effect. Notably, NS-2 simulations show that the number of rebroadcasts quickly drops close to its minimum value as the average timer duration is increased beyond a critical threshold. This phenomenon can be explained by noting that with high average timer duration redundant rebroadcasts caused by the delay of MAC layer do not occur with probability close to one. Thus, beyond a critical threshold for the random duration, the performance of the scheme is sufficiently close to the idealistic one with high probability. We call these timer settings the efficient regime of a timer-based scheme. We compute the performance in the efficient regime assuming idealistic MAC/PHY layer, thus leveraging the power of simplistic assumptions all while establishing the relevance of our findings via the notion of the efficient regime. Moreover, we explain some potential extensions to DRB and DCCB algorithm for making them even more robust and adaptive to the node failure or topology changes in Ad Hoc or sensor networks.

The structure of this paper is as follows. We describe existing timer-based schemes in Section II. We propose the DRB and DCCB schemes in Section III. In Section IV, we study and compare the performance and robustness of the timer-based schemes through simulations. We analyze the backbone size of DRB and DCCB schemes in Section V. In addition, we give local repair algorithms for DRB and DCCB schemes in Section VI for the network with topology changes. Finally we conclude the paper in Section VII.

\section{RELATED BROADCAST SCHEMES}

In this section we argue for our selection of broadcast schemes to which we compare to DRB and DCCB in Section IV. There exist only two deterministic timerbased broadcast schemes in the literature: Scalable Broadcast Algorithm (SBA) and the Stojemnovic scheme. Indeed, a timer-based approach brings about inherent elements of randomness which are easier to handle in a probabilistic scheme where full reachability does not have to be guaranteed. A comprehensive comparison of the entire host of existing probabilistic schemes is beyond the scope of the paper and would distract from the actual objective of demonstrating the benefits of a hybrid backbone. For a list of probabilistic schemes [2], [21], [22] and existing comparative work see [3], [4] and references therein. Area- and distance-based schemes [2], [21], e.g., use actual geographic information about 
the nodes which naturally changes the tradeoff game. Notably, counter- and color-based [22] schemes are probabilistic and timer-based but use only the explicit or implicit information provided by overheard broadcast packets. The more recent color-based schemes exhibit performance similar to the counter-based ones [22]. At last, a new scheme was proposed [23] most recently, where non-random timers are set depending on neighbor locations such as to optimize efficiency or latency. We do not include this scheme in our comparison since their timers are non-random.

In summary, we find it appropriate and useful to focus in this paper on the class of deterministic timerbased broadcast schemes and to add the counter-based broadcast as a representative probabilistic timer-based scheme for which a comparison is meaningful.

\section{A. SBA scheme}

The SBA scheme of Peng and Lu reduces the number of broadcast nodes as follows [6]. When a node $v$ receives a broadcast packet, instead of forwarding the packet immediately, it waits for a random time. Denote the set of neighbors of node $v$ by $N(v)$. For each of its neighbors $w$ that has forwarded the broadcast packet, node $v$ removes $w$ and $N(w)$ from $N(v)$. If the resulting set of nodes does not become empty after the random time, node $v$ forwards the broadcast node; otherwise node $v$ does not forward the packet.

\section{B. Stojmenovic scheme}

Stojmenovic proposed a timer-based scheme for broadcasting [10], [11] based on Wu and Li's marking process [5]. The scheme applies the geographic information, and uses only 1-hop information to implement the marking process. It calls the marked nodes "gateways" and the broadcast scheme only uses the gateways to forward the packet to all nodes in the network. In addition, it reduces the set of forwarding nodes further by running a neighbor elimination algorithm over the gateways, similar to the one used in SBA.

\section{Counter-based scheme}

The counter-based scheme is a simple probabilistic scheme [2]. In the counter-based scheme, when a node receives the broadcast packet for the first time it starts a random timer and counts how many times it overhears the same broadcast until expiration. If this number is less than some threshold $\eta$ then it rebroadcasts the packet. Clearly, a larger $\eta$ increases the probability of nodes rebroadcasting the packet and with it the reachability.
However, large values of $\eta$ also result in inefficiencies in term of number of rebroadcasts.

\section{DRB AND DCCB SCHEMES}

The major difference of our proposed schemes to existing ones lies in their hybrid broadcast backbone. In a nutshell, the proposed algorithms assume a given static Dominating Set (DS), respectively a Weakly Connected Dominating Set (WCDS) for which they build a set of connecting nodes for each broadcast on the fly. To this end, the algorithms employ a distributed marking algorithm. The idea of a hybrid backbone is novel. The same idea have been used in [30] to distribute the work load among nodes systematically for increasing the life time of sensor nodes.

\section{A. Background on Dominating Sets}

Many algorithms to build a WCDS have been proposed in the literature [27], [28], [31]-[33]. In some algorithms the generated WCDS based on an independent set. Consequently these algorithms keep the size of backbone at most a constant factor of the Minimum Connected Dominating Set (MCDS) [27], [28]. For example, the algorithm proposed by Wan, Alzoubi and Frieder build a WCDS using the minimum ID or the weights of the nodes on a rooted spanning tree, which is an independent set [28]. However, building and maintaining a near optimal WCDS is costly. Minor changes like failure or movement of a single WCDS node are not repairable locally, and require global reconstruction [34]. Note that finding an MCDS of a wireless network is an NP-complete problem [35], so such algorithms are used to estimate the MCDS.

The most common algorithms for constructing an efficient DS are clustering algorithms. These select a set of nodes as clusterheads. The set of clusterheads build an Independent Dominating Set (IDS) which is smaller than a constant factor of the MCDS size [24]-[26]. There are also some algorithms that build a Maximal Independent Set (MIS) [28], [36] (MIS is also a DS). However, some clustering algorithms does not build necessary an IDS [7], [31], [32].

\section{B. Dynamic Reflector Broadcast (DRB)}

The DRB algorithm assumes that a WCDS has been computed and that all network nodes know their WCDS neighbors. The nodes of the WCDS are called dominators; further, the network nodes which have more than one dominator as neighbor are called reflectors. The scheme works as follows. 


\section{Dynamic Reflector Broadcast}

1) The broadcast may be initiated by any node by simply broadcasting a packet to all its neighbors.

2) Any dominator that hears a particular broadcast packet for the first time rebroadcast the packet after a short delay. It also appends to the packet the list of all neighboring dominators (including itself) and removes any other appended list. A typical setting is a delay uniformly distributed in the interval $\left[0, T_{D}\right]$.

3) Any reflector that hears a particular broadcast packet for the first time starts a timer of random duration. A typical setting is a uniform distribution in the interval $\left[0, T_{R}\right]$ where $T_{R}$ is much larger than $T_{D}$. Until its timer expires the reflector listens to all rebroadcasts of the same packet and logs as "done" the sending dominators corresponding the target dominators listed in the packet. Upon expiration of the timer it checks whether any of its neighboring dominators is not logged as "done". If not, it takes no further action. If so, it rebroadcasts the packet to all its neighbors. It also appends to the packet the list of all its neighboring dominators and removes any other appended list, in order to inform all neighboring reflectors.

Some notes on parameter settings are in order. The timer for dominators $T_{D}$ helps avoiding collisions between dominators which may receive the broadcast within short time of each other. For a network with collision free MAC layer, $T_{D}$ can be set to zero. The timer for reflectors $T_{R}$ will be set depending on density of the reflectors, packet size and background traffic (see the in-depth discussion in Section IV).

For an illustration we refer to Fig 1 where the dominators are indicated by squares, the reflectors by red circles and the originating node by $S$. The packet may take different paths, depending on the realized timer values. For example, if $R 4$ happens to have the shortest timer among $R 2-R 5$, then the broadcast begins its path as $S \rightarrow D 3 \rightarrow R 4 \rightarrow D 1$. If even the timer of $R 1$ expires before the one of $R 2$ then the packet's path is $S \rightarrow D 3 \rightarrow R 4 \rightarrow D 1 \rightarrow R 1 \rightarrow D 2$ with the obvious bifurcation at $D 3: \ldots \rightarrow R 5 \rightarrow D 4 \rightarrow R 6 \rightarrow D 5$. Ideally, no other rebroadcasts occur; in particular, $R 2$ and $R 3$ will take no action, providing they are being informed of the broadcast state by the relay of $R 4 \rightarrow D 1$ and the broadcast of $D 2$, respectively, before their timers expire.

In other words, under the assumption of an ideal MAC layer, the broadcast will happen along a tree which is,

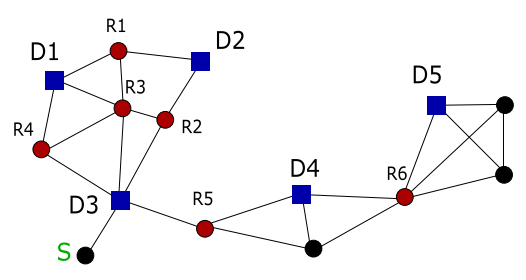

Fig. 1. Set of dominators and reflectors for DRB scheme. The broadcast packet can reach $D 2$ through the paths $D 3 \rightarrow R 2, D 3 \rightarrow$ $R 3 \rightarrow R 1$ and $D 3 \rightarrow R 4 \rightarrow D 1 \rightarrow R 1$.

however, random. In reality, MAC and physical layer consume time to transmit the packet due to background traffic and reflectors may broadcast redundant packets. To continue the above example (see Fig 1) if the timers of reflectors $R 3$ and $R 4$ end shortly after each other, the networking layers of both reflectors may hand the packet to their MAC layer for broadcast (to serve $D 1$ ) before hearing the other's rebroadcast. In Section IV we will study timer-based schemes with realistic MAC layer, and propose some techniques to avoid this cross-layer problem. Another important property of DRB is:

Lemma 1: In a network with lossless MAC layer, DRB reaches all nodes.

Proof: By design of DRB, the dominator of the originating node must eventually rebroadcast the packet. Also by design, the broadcast reaches the set of dominators which are within 2-hops of each other and which contains the originator's dominator. By definition of a WCDS, this set is the entire DS. Since, all dominators rebroadcast the packet once, all nodes are covered.

\section{Dynamic Connector-Connector Broadcast (DCCB)}

The DCCB algorithm assumes that a DS has been computed, whose members are again called dominators and that all network nodes know the dominators within 1 or 2 hops distance. Note that it is necessary to connect all dominators within 3 hops of each other in order to guarantee a CDS over a lossless MAC layer. The network nodes which have more than one dominator within at most 2 hops distance are called connector. The scheme works as follows.

\section{Dynamic Connector-Connector Broadcast}

1) Every network node $A$ compiles the lists of dominators that are exactly 1-hop and 2-hops away, denoted by $L_{1}(A)$ and $L_{2}(A)$ respectively. If $A$ is itself a dominator it adds itself to the list $L_{1}(A)$. Every node $A$ appends these lists $L_{1}(A)$ and $L_{2}(A)$ to the broadcast message in the packet when 
rebroadcasting it, removing any other appended lists.

2) When a dominator hears a broadcast packet for the first time it rebroadcasts the packet after a short random delay. In a typical setting, the delay is uniform in the interval $\left[0, T_{D}\right]$.

3) When a connector node $A$ first hears a packet $m$, it starts a random timer. In a typical setting the duration is uniform in the interval $\left[0, T_{C}\right]$ where $T_{C}$ is much larger than $T_{D}$. The connector generates new lists $L_{1}(A, m)$ and $L_{2}(A, m)$ specific to the packet $m$ which it initializes to $L_{1}(A)$ and $L_{2}(A)$ respectively.

4) Whenever a connector $A$ hears a broadcast of the packet $m$, say by node $B$, including the first time it hears $m$, it deletes all elements of $L_{1}(A, m)$ and $L_{2}(A, m)$ that belong to $L_{1}(B)$. Being in range of $B$, these dominators are dealt with. It also deletes from $L_{2}(A, m)$ any elements that belong to $L_{2}(B)$.

5) If at timer expiration, either $L_{1}(A, m)$ or $L_{2}(A, m)$ are non-empty, node $A$ rebroadcasts the packet.

Similarly as with DRB, the parameter $T_{D}$ can be set to zero for a network with collision free MAC layer. We consider random timers for dominator to avoid collisions between dominators which receive the broadcast packet shortly after each other. The parameter $T_{C}$ should be set depending on node density in the network, packet size and background traffic. We refer to the discussion in Section IV.

For a demonstration of the algorithm we refer to Fig. 2 which shows a set of dominators (squares) and connectors (pink circles), as well as the node $S$ which originates the broadcast. As with DRB, the broadcast packet may take several different paths according to which timers expire first, resulting in backbones that vary randomly according to the timer values as well as others random effects including transmission delay caused by the MAC layer and background traffic.

The need to connect dominators within 3 hops of each other (see, e.g., D2 and D3 in Fig. 2) leads to a source of inefficiencies not present with a WCDS. If $D 1$ receives the packet via $D 2 \rightarrow C 5 \rightarrow C 4$, e.g., then $C 2$ will still attempt to forward the packet to $D 1$, which is an example for redundant connector between 3-hop away dominators.

Again, an important property is easily established.

Lemma 2: In a network with lossless MAC layer, $D C C B$ reaches all nodes.

Proof: It is well known that connecting 2-hop and 3-hop away DS nodes (dominators) will result a CDS. The rest

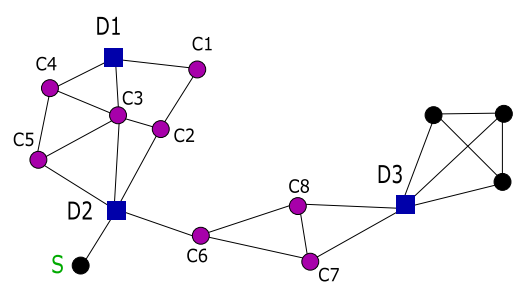

Fig. 2. Set of dominators and connectors in DCCB scheme. The broadcast packet can reach $D 1$ through the paths $D 2 \rightarrow C 3, D 2 \rightarrow$ $C 5 \rightarrow C 4$ and $D 2 \rightarrow C 2 \rightarrow C 1$

of the argument is completely analogous to lemma 1 .

To summarize or design choices, the hybrid nature of both DRB and DCCB are motivated combining two benefits, robustness from the dynamic part of the backbone, and efficiency from the static part. DRB is expected to perform particularly efficient due to its tree shaped backbone under idealistic MAC layer assumptions. DCCB, on the other hand, can relay on any DS and is expected to be particularly robust due to the larger set of connectors between dominators. In addition, generated DS by clustering algorithms are locally repairable [26]. Therefore, DCCB could show desirable performance in ad hoc networks with fast topology changes.

\section{Timer-BASEd Schemes In Simulation}

In this section we study the performance of different timer-based schemes for a homogeneous dense network by simulation. The metrics used are efficiency, i.e., number of rebroadcasts, latency, and reachability. We also address robustness, characteristics of timer-based schemes to be noted in simulation as well as a the potential for a cross-layer design to mitigate the effects of using timers on latency.

In all simulations we employ the NS-2.29 simulator [29]. Nodes are uniformly distributed in a $2400 \mathrm{~m}$ by $1800 \mathrm{~m}$ area and each node has the default radio range of $250 \mathrm{~m}$. We employ the IEEE $802.11 \mathrm{~b}$ MAC protocol with basic transmission rate $1 \mathrm{Mbps}$ and broadcast packet size of 125 Bytes. All results have been computed by averaging the performance over 200 realization for each scheme, by varying the originating node of broadcast randomly among the nodes. Packet size is kept constant and no background traffic is present in any simulation. Clearly, both factors increase the time from reception to eventual relaying of a broadcast packet. Though an in-depth study is beyond the scope of this paper, some first order valuable insight into the effect of these factors on performance could potentially be gained for timerbased schemes by conceptually absorbing the additional random delays into the timers. 


\section{A. Using Timers: Trading off Latency for Efficiency}

Using timers constitutes a clever mechanism to detect network topology on the fly with the potential for superior performance at the price of latency, as we will demonstrate. Less obviously, timer-based schemes perform less efficiently over realistic MAC and physical layers with non-zero packet transmission time, contention and collision than if these layers were idealistic.

Indeed, in a timer-based scheme, the network layer of the node may decide at timer expiration to rebroadcast the packet based on information collected from all overheard broadcasts of the same packet. It forwards the packet to the MAC layer where it is temporarily buffered. Before the MAC layer transmits the packet the node may obtain new information from further rebroadcasts which would reverse its decision to rebroadcast. However, in the absence of any cross-layer design, the broadcast scheme (network layer) cannot stop the MAC layer which results in a redundant transmission. The longer the waiting time in the MAC queue as compare to the network timer, the higher the chance for such redundancy. Clearly, the chance increases by adding to the volume of background traffic, using large broadcast packets, reducing the capacity and specially increasing density of the nodes.

Essentially, keeping all other factors constant, increasing the average of the network layer timer results in fewer redundant transmissions. Simulation results depicted in Fig. 3(a) confirm this intuition. We used 500 nodes with uniformly distributed timer, and varied the average timer length. The trade-off between the number of broadcast nodes and latency for all timerbased schemes is only too obvious, with very small timers essentially resulting in a blind flood. Note that each point on the plots shows the average number of rebroadcasts, reachability and latency of a timer-based scheme over 200 realizations.

\section{B. Efficient Regime}

While small time settings result practically in a blind flood according to Subsection IV-A, the number of rebroadcasts quickly drops close to its minimum value as the average timer duration is increased beyond a critical threshold. This is explained by the observation that redundancy caused by the lower layers occurs with probability practically zero, thus resulting in a performance close to that of an idealistic MAC/PHY layer. Note that the critical threshold depends on density, $\mathrm{MAC} / \mathrm{PHY}$ layer and background traffic in the network. We call timer settings that result number of rebroadcasts within an acceptable predefined tolerance of the one over idealistic MAC/PHY layer the efficient regime of a timerbased scheme. Typically, also the reachability is close to idealistic in the efficient regime.

The relevance of the efficient regime for theoretical work lies in establishing a regime of timer settings where the performance can be assessed within an acceptable tolerance by assuming idealistic MAC/PYS layer. In practice, an efficient regime with acceptable tolerance is found by simply letting the timer values be large enough such that the scheme performs beyond the distinct "kneepoint" in the performance plots (see Fig. 3(a)). The efficient regime provides also a reasonable setting of timer values for a meaningful empirical comparison of broadcast schemes.

A prominent finding of our simulations is that DRB uses fewer number of rebroadcasts than other timerbased schemes for any given average latency in the network. Note that this result does not change when we vary the packet size though we do not show these results here due to space limitations. Assuming settings in the efficient regime for the moment, the counter-based scheme with threshold $\eta=2$ is competitive, but has inferior reachability (see Fig. 3(b)). With threshold set to $\eta=3$, however, it is less efficient than DCCB and the Stojmenovic schemes. Finally, as we will show later in this section, as the density of nodes increases, DCCB becomes more efficient than the Stojmenovic scheme.

Outside their efficient regimes, i.e., for the small timer values with performance left of the "knee-points", the schemes use a large number of broadcasts (see Fig. 3(a), left part). The Stojmenovic scheme has less number of rebroadcasts than other schemes for very short timers, because it only uses subset of nodes (the "gateways") for broadcasting. In practice, the schemes must employ sufficiently large average timer values to ensure that they are efficient an issue which we address via a suggestion of a cross-layer at the end of the section.

\section{Impact of Node Density on Performance}

We next analyze how the performance of timer-based schemes in their efficient regimes depends on node density. To vary the density we change number of nodes in the area from 200 to 1200 . Fig. 3(c) indicates that the number of rebroadcasts for DRB and DCCB remains roughly constant as the density increases; in fact, it depends only on the area of homogeneous dense network [37]. In contrast, as our results indicate, the number of broadcast nodes increases with density for the SBA and Stojmenovic schemes. Our results also verify that the number of broadcast nodes in the counter-based schemes 
is bounded by a function of the area of the network as has been established in previous work [22]. For a comparison of performance see also Fig. 3(d).

\section{Robustness to Node Failure}

Though hardly addressed in the literature, node failure can be detrimental to broadcast schemes. Nodes can fail due to loss of power, being switched off, or malfunction. Here, we study the robustness of broadcasting schemes in terms of the average efficiency and the average reachability for different probabilities of node failure.

Static schemes with sparse backbones are particularly sensitive to the node failure which may cause a disconnection of part of the backbone and consequently a low reachability. Hence one way to improve robustness of static schemes is to trade it off against efficiency and to increase the redundancy of the backbone.

Being dynamic, timer-based schemes are quite robust to node failure. In a way, timer-based schemes enjoy the robustness of a static scheme with a very redundant backbone, but eventually only a small subset of these nodes get used.

We simulated a network of 500 nodes. Node failure is assumed to occur with equal probability $p$ for all nodes and independently of each other. Figs 3(e) and 3(f) compare the average efficiency and reachability of the schemes for different $p$. Notably, SBA achieves full reachability because it potentially includes all network nodes in the broadcast backbone, it will ensure that the packet reaches all nodes the network is connected.

DRB and DCCB both achieve excellent reachability. However, they cannot guarantee full-reachability like SBA because part of their backbones (the corresponding WCDS and DS) are predetermined before the broadcast and are not replaceable during a broadcast.

Counter-based schemes also enjoy good reachability. The Stojmenovic scheme, however, is less robust when compared to other schemes, because its potential backbone is smaller the potential backbone of other schemes.

\section{E. Cross-Layer Design to Guarantee Efficiency}

We finally propose a cross-layer design that allows timer-based schemes to perform in their efficient regimes even for very small average timer values. As we discussed in section IV-A, when the delay in the MAC queue is comparable to the average timer values used by the timer-based scheme at the network layer, a node may learn that no action is required of it only after handing the broadcast packet to the MAC layer, unable to stop the redundant transmission.
One solution is to give the network layer, and hence the broadcast scheme, the ability to annihilate packets that are present in the MAC queue. Alternatively, the MAC layer could be given the ability to check with the network layer whether to drop a broadcast packet just before transmitting the packet. The potential gains in terms of efficiency can be read off directly from Fig. 3(a): the knee-points moves to the left and the efficient regime of the schemes is reached for a very small latency.

\section{ANALYSIS: EFFICIENCY AND COMPLEXITY}

Among the performance metrics for broadcasting in wireless networks, its efficiency and overhead stand out for their relevance. Indeed, the number of transmissions directly impacts power consumption, bandwidth utilization, and latency. Using fewer nodes for broadcasting reduces the chance for contention and collision which waste resources and add to the latency. This fact has been proved analytically in [38]. The same paper shows also that a broadcast scheme has throughput within constant factor of broadcast capacity if and only if the backbone size is within a constant factor of MCDS. Here, we prove that DRB and DCCB backbones have this property in their efficient regimes.

Our analysis here builds on the following two idealistic assumptions ${ }^{1}$ which are at the basis of most existing analytical work in this field. We assume, first, that the MAC layer is collision free and, second, that the transmission time of packets is zero. While these assumptions are idealistic in general, we point out that in their efficient regimes and in the absence of background traffic timer-based schemes perform roughly as they would under the above stated first assumption (cpre. section IV). Indeed, large timer values or an appropriate cross-layer design would practically annihilate the chances for redundant rebroadcasts caused by transmission delays below the network layer.

In order to address the optimal performance of the schemes we restrict our attention to static (predetermined) portions of the backbones which are near optimal. More precisely, we impose that the DCCB scheme operates with a near optimal DS computed by a clustering algorithm [25], [26], and that the DRB scheme operates with a near optimal WCDS generated by a [28].

\footnotetext{
${ }^{1}$ We stress the fact, that our simulations did not impose these assumptions and employed the realistic representation of the MAC layer as implemented in ns-2.
} 
(a)

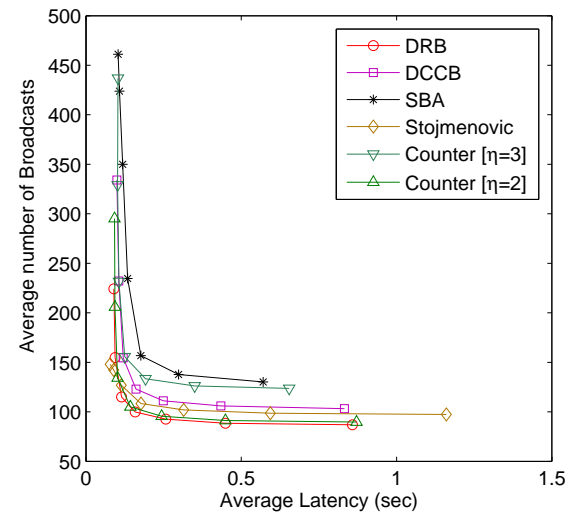

(b)
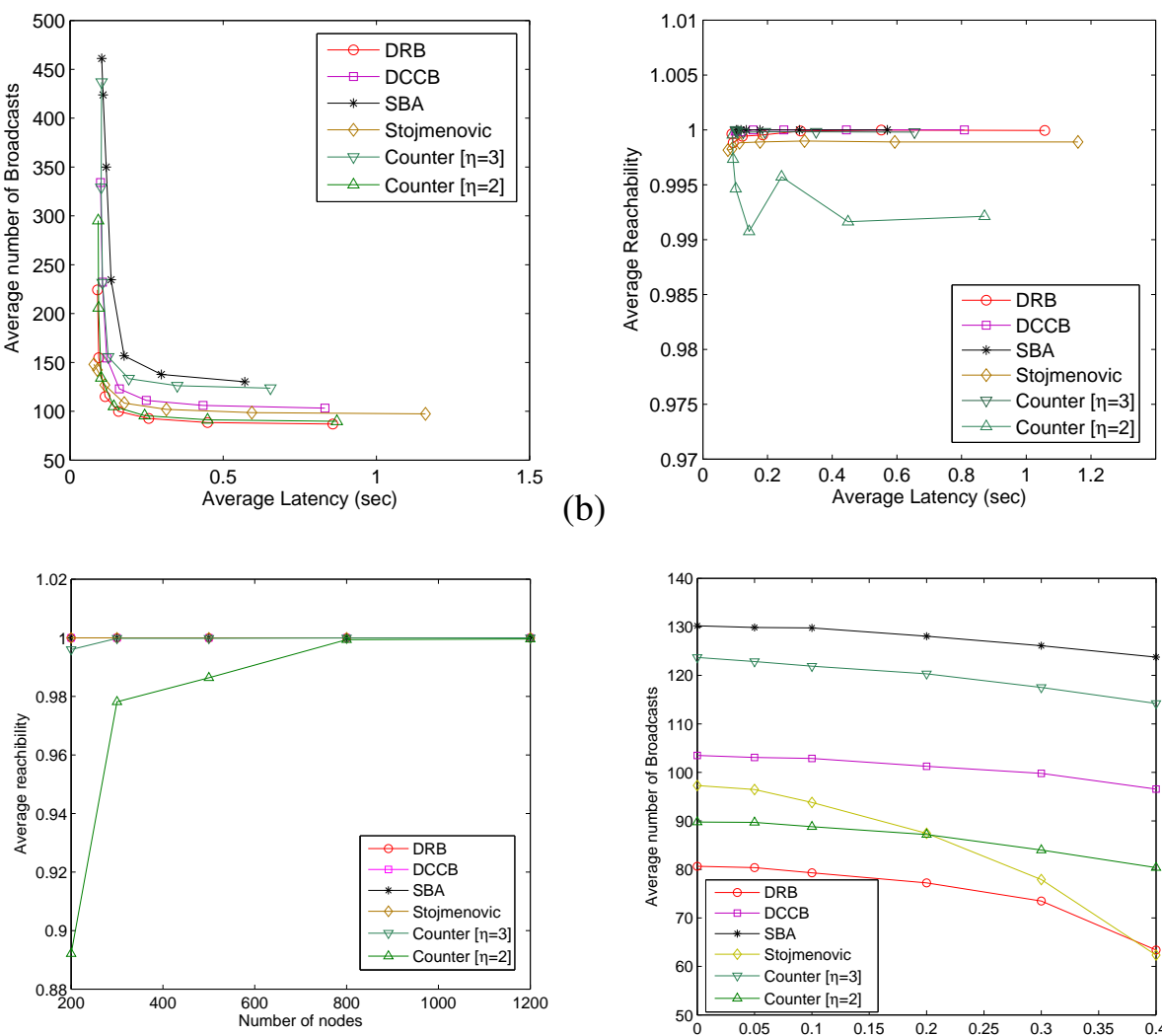

(d)

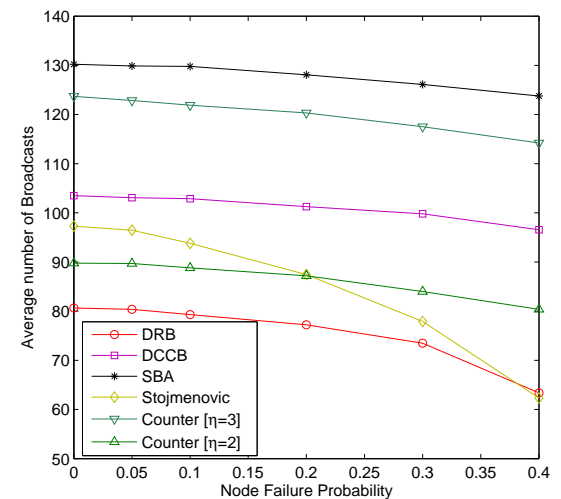

(e) (c)

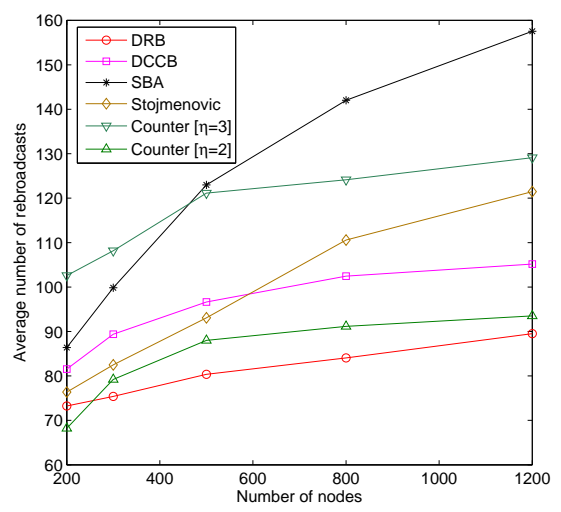

(f)

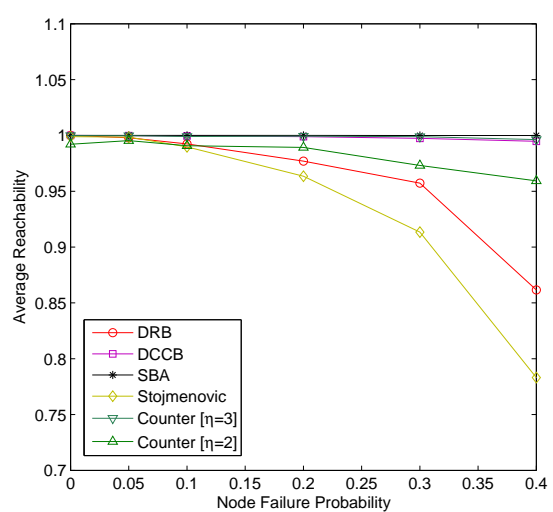

Fig. 3. (a,b) Efficiency and reachability of timer-based schemes against latency. (c,d) Efficiency and reachability in efficient regimes against node density (e,f) Efficiency and reachability in efficient regime against node failure probability

\section{A. Number of Rebroadcasts}

Denote the size of near optimal WCDS and DS as $N_{\text {WCDS }}$ and $N_{\text {DS }}$ respectively. Here, we compute the number of rebroadcasts of DRB and DCCB under the assumptions explained above in terms of $N_{\text {WCDS }}$ and $N_{\text {DS. }}$. We also compute the approximation factor which is the ratio of the number of rebroadcasts to that of the MCDS size. More details have been provided in the technical report of this work [37].

1) Number of rebroadcasts in DRB: We recall that for idealistic MAC/PHY layer the DRB broadcast algorithm guarantees that every reflector transmits only if the packet reaches a new dominator, so it uses at most $N_{\text {WCDS }}-1$ reflectors. For a more generally valid worst case bound on the approximation factor consider a dominator that fails or rebroadcasts the packet after a long delay. Then, several independent reflectors may try to forward the packet to it. However, still assuming the ideal MAC layer imposed on this section these reflectors can not be within range of each other, and their number is at most 5 for each dominator by simple geometry. Thus, the maximum number of rebroadcasts is
$(5+1) N_{\mathrm{WCDS}}=6 N_{\mathrm{WCDS}}$. Combining with the bound for the independent sets of [27] in terms of MCDS size the approximation factor is at most $4 * 6=24$.

2) Number of rebroadcasts in DCCB: For a generally valid worst case bound on the approximation factor consider all connectors which directly and indirectly forward the packet to the same dominator. However, still assuming the ideal MAC layer implies that direct connectors and indirect connectors build two independent sets. We can show by geometric arguments that there are at most 5 direct and 18 indirect connectors for each dominator. Thus the number of nodes used for broadcasting is at most $(1+5+18) N_{\mathrm{DS}}=24 N_{\mathrm{DS}}$. Again, from [27] the approximation factor is at most $4 * 24=96$.

\section{B. Complexity and Overhead}

First, we show that the length of the list which is appended to the broadcast packets is bounded. Since, dominators of near optimal WCDS or DS build an independent set, number of dominators in 1-hop away are 2- hop away from a fixed node is at most 5 and 18 respectively. So, the length of the appended list is at 
most $5 S$ in DRB and it is at most $(5+18) S$ in DCCB, where $S$ is the length of array which represent the ID of a dominator. Moreover, it shows that the amount of computation that is imposed to a node after hearing a broadcast packet is bounded (at most 5 comparisons in DRB and 23 comparisons in DCCB).

Second, we show that the number of overhearings of the same broadcast packet is bounded for every node. A node only hears the transmissions that target some dominators which are at most within 2 hops from the node in the DRB scheme, and within 3 hops in DCCB. The number of such dominators is less than 25 in DRB and 49 in DCCB [27]. Moreover, the schemes imply that no two neighbor nodes target the same dominator, which implies that the number of transmissions heard by a node and which target the same dominator is less than 5 . Therefore, the number of overhearings is less than $5 * 25$ in DRB and $5 * 49$ in DCCB.

From the two arguments above, we conclude that a broadcast packet imposes at most $O(1)$ computation to every node and therefore the total computation of the nodes is $O(n)$, where $n$ is the number of nodes. Note that here we do not consider the complexity of building a near optimal WCDS or DS. It has been shown that the complexity of the WCDS built by the algorithm proposed in [28] is $O(n \log (n))$ and the DS build by the algorithm proposed in [25] is $O(n)$.

\section{EXTENSIONS TO DRB AND DCCB ALGORITHMS}

The ability of dynamic nodes to join the broadcast backbone voluntarily give DRB and DCCB the capability to adapt to the scarceness of resources and to repair node failure. We develop these ideas in the following not with an eye on quantifying savings but to demonstrate the potential of these extensions.

\section{A. Adaptivity to Scarcity of Resources}

We assign a weight to each node that reflects its degree of connectivity, power consumption, or mobility and allow reflectors (connectors in DCCB) with higher priority to join the DRB backbone during broadcast. This is accomplished by shortening the random back-off timing for those nodes.

\section{B. Repair Mechanism to Counter Node Failure}

As we explained in Section III-C, DCCB scheme guarantees full-reachability on any DS, and some clustering algorithms are robust against topology changes in the network [25], [26]. So DCCB is applicable for the networks with node failure and even mobility.
The DRB broadcast is also robust to node failure, providing very high reachability even with non-negligible node failure, thanks to its use of a dynamic reflectors. In order to strive for full-reachability in the event of node failure, we propose a repair algorithm for DRB.

The suggested DRB-repair algorithm builds on periodic messages from all nodes. Every nodes sends a "Hello" message periodically and transmit its ID and a list of its dominators. As a consequence, all nodes will be aware about the dominators which are in 1-hop or 2-hops away and have not failed.

The DRB scheme would fail to disseminate the broadcast packet only when a reflector which is the only reflector between a pair of dominators or when a dominator fails. In this case, the DRB-repair algorithm asks from the neighbor nodes of the failed node to rebuild a DS locally and to rebroadcast under the DCCB scheme (more details in [37]). Note that the DRB-repair algorithm repairs the WCDS backbone locally. However, in some cases local repair algorithm can not fix a WCDS [34]. So, the algorithm does not grantee full-reachability.

\section{CONCLUSIONS}

The contribution of this paper is two-fold. First, we introduce two novel deterministic timer-based schemes: DRB and DCCB. Both possess an efficiency within a factor of the optimum, a property which other deterministic timer-based schemes do not share and which we establish both analytically and in simulation. The principal distinguishing feature of DRB and DCCB from other existing timer-based schemes is their hybrid backbone consisting of a fixed and a variable part.

Second, we study by simulation the effect of the settings of the random timer for existing and proposed deterministic timer-based schemes. To the best of our knowledge, this issue has not been addressed in the literature before. We showed how the performance of these scheme depends on the random timer. Simulations not shown due to space constraints indicate that performance also depends on the broadcast packet size, the capacity of the wireless channel, the density of the nodes as well as the volume of background traffic.

The simulations presented demonstrate that DRB and DCCB have better reachability than counter-based probabilistic scheme. Also, they use a smaller number of nodes when compared to deterministic timer-based schemes. We proposed a simple cross-layer design which helps the timer-based schemes to work close to their maximal efficiency without sacrificing latency. Notably, combining analysis and empirical estimates of geometric efficiency 
constants we establish that close to their maximal efficiency DRB and DCCB operate within a constant factor of the optimum, provided that the given WCDS and DS have sizes within small constant factor of the optimum.

\section{ACKNOWLEDGMENT}

Financial support comes in part from NSF, grant number ANI-0338856, and from Texas ATP, project number 003604-0036-2003.

\section{REFERENCES}

[1] X. Hong, K. Xu, and M. Gerla, "Scalable routing protocols for mobile ad hoc networks," IEEE Network, vol. 16, pp. 11-21, 2002.

[2] S.-Y. Ni, Y.-C. Tseng, Y.-S. Chen, and J.-P. Sheu, "The broadcast storm problem in a mobile ad hoc network," in MobiCom, 1999, pp. 151-162.

[3] B. Williams and T. Camp, "Comparison of broadcasting techniques for mobile ad hoc networks," in MobiHoc, 2002.

[4] W. Lou and J. Wu, Localized Broadcasting in Mobile Ad Hoc Networks Using Neighbor Designation. in Mobile Computing Handbook, CRC Press, 2005, ch. 28.

[5] J. Wu and H. Li, "On calculating connected dominating set for efficient routing in ad hoc wireless networks," in DIALM, 1999, pp. 7-14.

[6] W. Peng and X.-C. Lu, "On the reduction of broadcast redundancy in mobile ad hoc networks," in MobiHoc, 2000.

[7] P. Sinha, R. Sivakumar, and V. Bharghavan, "Enhancing ad hoc routing with dynamic virtual infrastructures," in INFOCOM, 2001.

[8] W. Lou and J. Wu, "On reducing broadcast redundancy in ad hoc wireless networks," IEEE Transactions on Mobile Computing, vol. 1, no. 2, pp. 111-123, 2002.

[9] A. Qayyum, L. Viennot, and A. Laouiti, "Multipoint relaying for flooding broadcast messages in mobile wireless networks," in HICSS, vol. 9. IEEE Computer Society, 2002, p. 298.

[10] I. Stojmenovic, M. Seddigh, and J. Zunic, "Dominating sets and neighbor elimination-based broadcasting algorithms in wireless networks," IEEE Trans. Parallel Distrib. Syst., vol. 13, no. 1, pp. 14-25, 2002.

[11] I. Stojmenovic, "Comments and corrections to 'dominating sets and neighbor elimination-based broadcasting algorithms in wireless networks'," IEEE Trans. Parallel Distrib. Syst., vol. 15, no. 11, pp. 1054-1055, 2004.

[12] B. Chen, K. Jamieson, H. Balakrishnan, and R. Morris, "Span: an energy-efficient coordination algorithm for topology maintenance in ad hoc wireless networks," ACM Wireless Networks Journal, vol. 8, no. 5, pp. 481-494, 2002.

[13] J. Wu and F. Dai, "A generic distributed broadcast scheme in ad hoc wireless networks," IEEE Trans. Comput., vol. 53, no. 10, pp. 1343-1354, 2004.

[14] T. J. Kwon and M. Gerla, "Efficient flooding with passive clustering (pc) in ad hoc networks," SIGCOMM Comput. Commun. Rev., vol. 32, no. 1, pp. 44-56, 2002.

[15] W. Lou and J. Wu, "A cluster-based backbone infrastructure for broadcasting in manets," in IPDPS, 2003.

[16] J. Wu and W. Lou, "Forward-node-set-based broadcast in clustered mobile ad hoc networks," in Wireless Communications and Mobile Computing, vol. 3, 2003, pp. 155-173.

[17] W. Lou and J. Wu, "Double-covered broadcast (dcb): A simple reliable broadcast algorithm in manets," in INFOCOM, 2004.
[18] G. Calinescu, I. I. Mandoiu, P.-J. Wan, and A. Z. Zelikovsky, "Selecting forwarding neighbors in wireless ad hoc networks," Mob. Netw. Appl., vol. 9, no. 2, pp. 101-111, 2004.

[19] Z. J. Haas, J. Y. Halpern, and E. L. Li, "Gossip based ad-hoc routing," CoRR, 2002.

[20] J. Cartigny and D. Simplot, "Border node retransmission based probabilistic broadcast protocols in ad-hoc networks," in HICSS, 2003.

[21] Y.-C. Tseng, S.-Y. Ni, and E.-Y. Shih, "Adaptive approaches to relieving broadcast storms in a wireless multihop mobile ad hoc network," in IEEE Transactions on Computers, vol. 52, no. 5, 2003, pp. 545-557.

[22] A. Keshavarz-Haddad, V. Ribeiro, and R. Riedi, "Color-based broadcasting for ad hoc networks," in WiOpt, 2006.

[23] M. Heissenbüttel, T. Braun, M. Wälchli, and T. Bernoulli, "Optimized stateless broadcasting in wireless multi-hop networks," in INFOCOM, 2006.

[24] D. J. Baker and A. Ephremides, "The architectural organization of a mobile radio network via a distributed algorithm," IEEE Trans. Comm., vol. COM-29, no. 11, pp. 1694-1701, 1981.

[25] C. R. Lin and M. Gerla, "Adaptive clustering for mobile wireless networks," IEEE Journal on Selected Areas in Communications, vol. 15, no. 7, pp. 1265-1275, 1997.

[26] S. Basagni, "Distributed clustering for ad hoc networks," in ISPAN. IEEE Computer Society, 1999, pp. 310-315.

[27] K. Alzoubi, P.-J. Wan, and O. Frieder, "Weakly-connected dominating sets and sparse spanners in wireless ad hoc networks," in ICDCS. IEEE Computer Society, 2003, pp. 96-104.

[28] P.-J. Wan, K. Alzoubi, and O. Frieder, "Distributed construction of connected dominating set in wireless ad hoc networks," Mob. Netw. Appl., vol. 9, no. 2, pp. 141-149, 2004.

[29] S. McCanne and S. Floyd, "ns Network Simulator," http://www.isi.edu/nsnam/ns/.

[30] W. S. Conner, J. Chhabra, M. Yarvis, and L. Krishnamurthy, "Experimental evaluation of synchronization and topology control for in-building sensor network applications," in WSNA, 2003, pp. 38-49.

[31] Y. P. Chen and A. L. Liestman, "Approximating minimum size weakly-connected dominating sets for clustering mobile ad hoc networks," in MobiHoc, 2002, pp. 165-172.

[32] — "A zonal algorithm for clustering ad hoc networks," International Journal of Foundations of Computer Science, vol. 14, no. 2, pp. 305-322, 2003.

[33] D. Dubhashi, A. Mei, A. Panconesi, J. Radhakrishnan, and A. Srinivasan, "Fast distributed algorithms for (weakly) connected dominating sets and linear-size skeletons," J. Comput. Syst. Sci., vol. 71, no. 4, pp. 467-479, 2005.

[34] Y. P. Chen and A. L. Liestman, "Maintaining weakly-connected dominating sets for clustering ad hoc networks," Ad Hoc Networks, vol. 3, no. 5, pp. 629-642, 2005.

[35] M. V. Marathe, H. Breu, H. B. Hunt III, S. S. Ravi, and D. J. Rosenkrantz, "Simple heuristics for unit disk graphs," Networks, vol. 25, pp. 59-68, 1995.

[36] F. Kuhn, T. Moscibroda, T. Nieberg, and R. Wattenhofer, "Fast deterministic distributed maximal independent set computation on growth-bounded graphs," in DISC, 2005, pp. 273-287.

[37] A. Keshavarz-Haddad, V. Ribeiro, and R. Riedi, "DRB and DCCB: Efficient and robust dynamic broadcast for ad hoc and sensor networks," Rice University, Department of Statistics, Tech. Rep. TR-2006-09, 2006.

[38] _ , "Broadcast capacity in multihop wireless networks," in MobiCom, 2006. 